\title{
Precarização do trabalho em saúde e o sofrimento mental no Brasil no contexto da COVID-19
}

\author{
Precarious work in health and mental suffering in Brazil within the context of COVID-19 \\ Trabajo precário em salud y sufrimiento mental en Brasil con el contexto de COVID-19
}

Recebido: 22/03/2021 | Revisado: 29/03/2021 | Aceito: 31/03/2021 | Publicado: 10/04/2021

\author{
Israel Coutinho Sampaio Lima \\ ORCID: https://orcid.org/0000-0002-1929-6142 \\ Universidade Estadual do Ceará, Brasil \\ E-mail:israel.coutinho@aluno.uece.br \\ Cidianna Emanuelly Melo do Nascimento \\ ORCID: https://orcid.org/0000-0001-5477-4413 \\ Universidade Estadual do Ceará, Brasil \\ E-mail: profa.cidianna.melo@gmail.com \\ Carla Barbosa Brandão \\ ORCID: https://orcid.org/0000-0002-6608-488X \\ Universidade Estadual do Ceará, Brasil \\ E-mail:carla.barbosa@aluno.uece.br \\ José Edmilson Silva Gomes \\ ORCID: https://orcid.org/0000-0003-0688-2254 \\ Universidade Estadual do Ceará, Brasil \\ E-mail:edmilson.gomes@aluno.uece.br \\ José Jackson Coelho Sampaio \\ ORCID: https://orcid.org/0000-0003-4364-524X \\ Universidade Estadual do Ceará, Brasil \\ E-mail: jose.sampaio@uece.br \\ Antonio Rodrigues Ferreira Júnior \\ ORCID: https://orcid.org/0000-0002-9483-8060 \\ Universidade Estadual do Ceará, Brasil \\ E-mail: arodrigues.junior@uece.br
}

\begin{abstract}
Resumo
O presente estudo teve como objetivo refletir sobre as repercussões da precarização do trabalho em saúde e o sofrimento mental enfrentado por profissionais da saúde, no contexto da pandemia da COVID-19 no Brasil. Síntese de conteúdo: trata-se de um ensaio teórico crítico, realizado a partir da problematização da Medida Provisória 927 de 2020, aplicada ao contexto da pandemia da COVID-19, que tem resultado na superexploração do trabalhador da saúde. Compreende-se que o desmonte das leis trabalhistas tem ocorrido de modo sistemático e difuso no Brasil, porém, nos últimos três anos, essa vertente tem se intensificado, encontrando no contexto da pandemia pelo novo coronavírus espaço para se estabelecer como meio legítimo. É a partir desta ação do Governo Federal que a flexibilização dos contratos trabalhistas, o aumento da jornada de trabalho, a redução do tempo de descanso e desobrigação sobre a segurança e a saúde ocupacional se estabelecem como meio legal. Isso acarreta o agravamento da precarização e da sobrecarga do trabalho, resultando em sofrimento mental pelos profissionais da saúde. Conclusão: torna-se fundamental ampliar o debate o peso da legislação trabalhista, portanto de suas reformas, sobre a saúde do trabalhador, o que impacta sobre a prática das entidades de classe e dos órgãos trabalhistas em prol da proteção dos direitos sociais e da preservação da saúde mental dos trabalhadores.
\end{abstract}

Palavras-chave: Riscos ocupacionais; Estresse psicológico; Pessoal de saúde; Legislação trabalhista.

\begin{abstract}
The present study aimed to reflect on the repercussions of precarious work in health and the mental suffering faced by health professionals in the context of the pandemic of COVID-19 in Brazil. Content synthesis: this is a critical theoretical essay, based on the problematization of Provisional Measure 927 of 2020, applied to the context of the COVID-19 pandemic, as a way to legitimize the overexploitation of the health worker. Thus, it is understood that the dismantling of labor laws is something historical in Brazil. However, in the last three years, this aspect has intensified, finding in the context of the pandemic by the new coronavirus space to establish itself as a legitimate means. It is from this new onslaught by the Federal Government that the flexibilization of labor contracts, the increase in working hours, the reduction of rest time and release on occupational safety and health are established as a legal means. This worsens the precarious work and mental suffering of health professionals, due to the lack of recognition and work overload. Conclusion: it is essential to expand this debate in view of the negligence of the Brazilian Government,
\end{abstract}


making it necessary new ways of reinventing class entities and labor bodies in order to protect social rights and preserve workers' mental health.

Keywords: Occupational risks; Stress psychological; Health personal; Legislation labor.

\section{Resumen}

El presente estudio tuvo como objetivo reflexionar sobre las repercusiones del trabajo precário em salud y el sufrimiento mental que enfrentanlos profesionales de la salud enel contexto de la pandemia de COVID-19 en Brasil. Síntesis de contenido: este es um ensayo teórico crítico, basado em la problematización de la Medida Provisional 927 de 2020, aplicada al contexto de la pandemia COVID-19, como una forma de legitimar la sobre explotación del trabajador de la salud. Por lo tanto, se entiende que el desmantelamiento de las leyes laborales es algo histórico en Brasil. Sin embargo, em los últimos tresaños, este aspecto se ha intensificado, encontrando enel contexto de la pandemia por elnuevoespacio de coronavirus para establecerse como unmedio legítimo. Es a partir de esta nuevaembestidadelGobierno Federal que laflexibilización de los contratos laborales, el aumento de las horas de trabajo, lareduccióndeltiempo de descanso y laliberación de laseguridad y saludeneltrabajo se establecen como unmedio legal. Estoempeoraeltrabajoprecario y elsufrimiento mental de los profesionales de la salud, debido a la falta de reconocimiento y sobrecarga de trabajo. Conclusión: es esencial expandir este debate en vista de lanegligenciadelGobiernobrasileño, haciendonecesarionuevas formas de reinventar las entidades de clase y los cuerpos laborales para proteger los derechos sociales y preservar la salud mental de los trabajadores.

Palabras clave: Riesgos laborales; Estrés psicológico; Personal de salud; Legislación laboral.

\section{Introdução}

O contexto da crise na saúde pública mundial, em geral, e brasileira, em particular, gerada pela pandemia da COVID19 proporcionou condições para as forças políticas de apoio ao atual Governo brasileiro aprovarem a Medida Provisória-MP No 927/2020 (Brasil, 2020), evidenciando ação que fragiliza a proteção trabalhista embutida na Carta Magna de 1988, o exacerba a exploração e impacta fortemente sobre a saúde dos trabalhadores, simultaneamente vulneráveis à pandemia.

Essa medida vem repercutindo na vida laboral e psicossocial dos trabalhadores da saúde, devido ao esgotamento profissional, por meio de aumento da jornada de trabalho, redução do tempo de descanso, redução das já baixas remunerações e suspensão de exigências sobre segurança e saúde ocupacionais, no que concerne o fornecimento e treinamento para uso dos Equipamentos de Proteção Individual-EPI (Gold, 2020), próprios de cada ramo de produção, acrescidos dos exigidos pela situação de pandemia viral.

Esse processo de precarização do trabalho vem se delineando de forma mais prática, desde as mudanças vividas no cenário mundial, com a crise do capital e da recessão econômica de 2008. Período este caracterizado pela desaceleração da segunda maior economia do mundo, a China, além de outros países (Andrade, Casulo \& Alves, 2018).

No Brasil, esta mesma crise econômica pouco foi sentida naquele momento. Porém vem repercutindo politicamente desde 2016, quando o governo do Partido dos Trabalhadores (PT), representado pela Presidente, foi deposto. O Vicepresidente, que assumiu após a deposição, membro do Partido do Movimento Democrático Brasileiro-MDB, deu início ao desmonte dos direitos trabalhistas conquistados nos anos anteriores, por sua vez, herdeiros em parte da Consolidação das Leis Trabalhistas-CLT, oriundos da Era Getúlio Vargas (Galvão \& Marcelino, 2018).

Neste ínterim, a maior precarização do trabalho toma forma concreta diante da flexibilização dos direitos trabalhistas e do enfraquecimento das organizações sindicais, sendo um dos pontos centrais da agenda política do Governo Federal no último triênio (Andrade, Casulo \& Alves, 2018).

As ofensivas sobre os direitos sociais e trabalhistas estão sendo agravadas (Galvão et al, 2019), inclusive pela execução da MP No 927/2020, nascida com o intuito de formalizar o estado de calamidade pública decorrente da COVID-19 (Brasil, 2020), Essa medida é duramente criticada pelo Conselho Federal de Enfermagem-COFEN, por meio de texto que expõe a situação do trabalho dos trabalhadores da Enfermagem, o que se aplica, em grande escala, para os demais trabalhadores da saúde (Cofen, 2020). A referida MP desrespeita a Constituição de 1988, por prejudicar direitos adquiridos, e a Norma $\mathrm{N}^{\circ}$ 155, da Organização Internacional do Trabalho-OIT, da qual o Brasil é signatário. A Norma da OIT afirma ser 
necessária a reavaliação periódica da política nacional, mas de modo a identificar mudanças na realidade social e garantir a preservação da saúde e da segurança ocupacionais, não de reduzi-las (Cofen, 2020; Cut, 2020).

Caracterizado o contexto do lançamento da referida MP e a crítica desenvolvida por órgãos de classe, o presente manuscrito busca problematizar o tema do aprofundamento da precarização do trabalho em saúde, considerando a circunstância da pandemia como justificativa para maior precarização de todo trabalho, destaque para o trabalho em saúde. Quais questões movem o raciocínio: Como a precarização do trabalho, em tempos de pandemia causada pela COVID19, vem sendo legitimada pela MP No 927/2020? Quais são as repercussões da MP No 927/2020 na saúde mental desses profissionais?

\section{Metodologia}

Trata-se de um ensaio teórico, o qual busca dar vida a relação do vir-a-ser, que constitui o sujeito e o objeto diante da relação da objetividade e subjetividade que envolve os fenômenos (Meneghetti, 2011). Logo, esta reflexão crítica busca problematizar o sofrimento mental vivido pelos profissionais da saúde no contexto da pandemia causada pela COVID-19 e a publicação da MP 927 de 2020, enquanto política pública que envolve a precarização do trabalho em saúde no cenário brasileiro. Reflexões estas ancoradas na literatura produzida sobre a temática, nas discussões realizadas durante as aulas do Programa de Pós-graduação em Saúde Coletiva, além das experiências práticas enquanto profissionais atuantes no enfrentamento da Sars-CoV-2, sejam em ações que vão desde a produção de conhecimento e de cuidados na área.

\section{Resultados e Discussão}

\subsection{O percurso para a legitimação da precarização do trabalho no Brasil}

O percurso histórico que vem legitimando o aprofundamento da precarização do trabalho no Brasil ficou fortemente evidenciado a partir de 2015, quando o resultado eleitoral e as práticas de gestão pública foram questionados, em clima de acirramento político-ideológica por meio do fortalecimento de oposição partidária situada no espectro ideológico da direita ou de uma postura simplificada como anti-Partido dos Trabalhadores-PT (Galvão \& Marcelino, 2018).

A grave crise política e econômica vivida pelo Brasil, nos últimos anos, em ciclos de piora e melhora, acompanha os ciclos internacionais com problemas próprios, em período que remonta ao fim da Ditadura Militar. Naquele momento, a inquietação sócio-econômica e o aumento da pobreza ofereceram condições ao fim da Ditadura. Hoje, a inquietação, o aumento da pobreza e a retomada mundial da hegemonia de uma direita neoliberal oferecem condições para graves prejuízos ao mais longo período de crescimento econômico, estabilidade política e experiência democrática vividas pelo país, iniciado em 1985.

Houve a recessão mundial de 1973-1975, a propalada década perdida dos anos 1980, a recessão mundial de 2008, sentida no Brasil a partir de 2014, e a mais nova crise político-econômica mundial gerada pela competição comercial USA x China, tanto quanto pela volatilidade do capital especulativo, agravados pela primeira pandemia do mundo globalizado, a da COVID-19 (Andrade, Casulo \& Alves, 2018).

Além do temor generalizado em decorrência direta do avanço da pandemia, as tensões psicossociais decorrentes das práticas preventivas de isolamento e não aglomeração, dos constrangimentos do enfrentamento político-ideológico entre os favoráveis às medidas de controle sanitário, embora com impacto econômico, contra os negacionistas, soma-se a crise decorrente do impacto econômico negativo, gerando desemprego e, a pretexto de evitar desemprego, a implantação das medidas de flexibilização que atingem os direitos sociais dos trabalhadores, em contradição com a razoável proteção trabalhista e de organização sindical até então garantidas pela Constituição Brasileira de 1988 (Druck, Dutra \& Silva, 2019). Isso se deve pelo entendimento que o capitalismo neoliberal, enquanto política global, consolidado no ideário da flexibilização 
dos contratos, em oposição à lógica fordista hegemônica anteriormente, estrutura-se a partir do desprezo pelo emprego formal (Pochmann, 2020).

$\mathrm{Na}$ era fordista, o trabalho formal era almejado pelos trabalhadores como forma de ascensão social. Tendo na estabilidade empregatícia condições que valorizavam o trabalho, a partir do modo de regulação rígida, dentro das leis trabalhistas de produção, as quais garantiriam uma "vida boa". Desta forma, os trabalhadores se agrupavam de acordo com sua classe profissional, por meio de entidades sindicais, pactuando contratos coletivos, como salários, tempo de serviço e jornada de trabalho, com base nos direitos sociais e previdenciários (Andrade, Casulo \& Alves, 2018; Galvão et al, 2019).

Com o avanço do capitalismo globalizado, pautado, no campo produtivo, pelo toyotismo, mas sobretudo pautado pela hegemonia do capital financeiro, a flexibilização da força de trabalho ganha força em governos liberais. Com a vida dita moderna, pelo surgimento das novas tecnologias, em um novo mundo, o trabalho toma características "full time", nas quais o trabalhador sempre está à disposição do empregador. Tornando o então trabalhador um colaborador, que deve ser resiliente diante da superalienação determinada pela supervalorização do capital variável (Andrade, Casulo \& Alves, 2018). Nesta lógica, a lucratividade das empresas passa a depender exclusivamente do colaborador, o qual jamais fará parte do capital.

No período 2003-2015, as manobras que buscavam pôr fim à Consolidação das Leis do Trabalho-CLT, a partir de sua flexibilização, encontraram resistência dos trabalhadores e de seus representantes, respaldados pelo Executivo Federal e flutuante maioria parlamentar. A partir do impeachment da presidente Dilma Rousseff ganhou potência a agenda neoliberal, com sucessiva aprovação de novos marcos legais, danosos ao trabalhador (Andrade, Casulo \& Alves, 2018).

Em 2016, por iniciativa do Executivo, o Parlamento Federal congelou os gastos públicos na área social por 20 anos, por meio da Emenda Constitucional-EC N ${ }^{\circ}$ 95. Logo em seguida, em 2017, a CLT sofreu nova flexibilização pela aprovação da Lei $n^{\circ} 13.429$, que permite a terceirização irrestrita do trabalho e da produção, além da Lei no 13.467 que altera a CLT a fim de adequar a legislação às novas relações de trabalho (Druck, Dutra \& Silva, 2019; Lourenço et al, 2019; Menezes, Moretti \& Reis, 2020).

Com tais normativas, a precarização do trabalho passa a ser regulamentada e aceita. Em consequência, um quantitativo importante de medidas desregulatórias do mercado de trabalho vêm sendo implementadas rapidamente. Situação agravada com a eleição presidencial de 2019, ganha por uma plataforma política mista de conservadorismo e intervenção pública nos comportamentos individuais e de agenda econômica ultraliberal, que vem acelerando a precarização do trabalho. Sobretudo quando se considera a extinção do Ministério do Trabalho, criado por Getúlio Vargas em 1930, portanto existente e operativo durante 88 anos (Andrade, Casulo \& Alves, 2018; Druck, Dutra \& Silva, 2019; Pochmann, 2020).

Por meio da MP No 873, de 2019, operacionaliza-se verdadeira cisão na organização coletiva do trabalho, no Brasil, pelo enfraquecimento do financiamento sindical. Impõe-se uma série de entraves de natureza administrativa, com forte teor burocrático, sobre o recolhimento da contribuição sindical, o qual deve ser autorizado individualmente pelo interessado, proibindo o desconto em folha de pagamento a ser pago por meio de boleto individual enviado para a residência do trabalhador. Tal lógica se opõe aos mecanismos de autorização por assembleia ou negociação coletiva, já histórica no movimento sindical (Andrade, Casulo \& Alves, 2018; Druck, Dutra \& Silva, 2019).

O processo de precarização do trabalho acelera-se por meio das novas modalidades de contratação, das alterações da jornada laboral, das formas de remuneração, das condições ocupacionais, do enfraquecimento do movimento sindical e da Justiça do Trabalho (Galvão et al, 2017; Galvão \& Marcelino, 2018)

Assim, a atual legislação trabalhista orienta-se para a redução do custo trabalho e para a superexploração do trabalhador, por meio de legitimação da carga horária de doze horas de trabalho por trinta e seis de descanso, negociação do banco de horas entre empregado e empregador, parcelamento das férias, redução dos intervalos intrajornada, redução do 
horário de almoço de uma hora para trinta minutos, prevalência do negociado sobre o legislado, contratação intermitente, terceirização de todos os setores e o fim da rescisão por comum acordo (Galvão et al, 2019).

Uma reflexão sobre a conjuntura política e econômica, mesmo rápida e panorâmica, focada na legislação, expõe que as manobras contra a proteção social, garantida até então pela CLT, só foram possíveis devido à convergência de interesses político-econômicos, o que gerou cenário favorável ao desmonte dos direitos trabalhistas. Diante disso, os trabalhadores brasileiros foram oprimidos por um cenário que desdobrou para dentro do país o clima econômico internacional, caracterizado pela guerra comercial USA x China e pela hegemonia da especulação financeira globalizada, representado no território nacional, como sintoma agregado de dependência, pelas votações desprovidas de apoio popular realizadas pelo Congresso Nacional, chanceladas pelo Poder Judiciário (Passos \& Lupatini, 2020).

\subsection{A nova ofensiva sobre a segurança e a saúde ocupacional dos trabalhadores da saúde}

A grave crise na saúde pública brasileira gerada pela COVID-19, diante da fragilidade do processo de sucateamento e desmonte de outra grande política pública arrojada, que é o Sistema Único de Saúde-SUS, vem servindo de palco para manobras governamentais que ferem diretamente a segurança e saúde ocupacional dos trabalhadores da saúde. É a partir da dramática situação gerada pela pandemia da COVID-19 que o Governo brasileiro está abrindo caminho para a passagem da "boiada", enquanto todos se preocupam com as consequências geradas pela nova cultura política das fake news pelas redes sociais e pelo novo coronavírus na saúde da população (Sintaema, 2020).

A MP N $\mathrm{N}^{\circ}$ 927, de 2020 (Brasil, 2020) traz para o cenário brasileiro alternativas trabalhistas ao enfrentamento do estado de calamidade gerada pela COVID-19. Mas a questão é: De quais alternativas o Governo Federal está falando? Quem seria prejudicado ou beneficiado por esta MP? Apesar da referida MP declarar em seu preâmbulo que as medidas elencadas ajudariam na preservação do emprego e da renda, o seu texto fragiliza o bem-estar psicossocial do trabalhador em prol do acúmulo de renda pelos empregadores.

As Centrais Sindicais e os juristas do trabalho entendem que as MPs anteriormente destacadas ferem a Carta Magna de 1988, por impedir que os sindicatos atuem na garantia dos direitos trabalhistas, por meio da ação coletiva (Cut, 2020). Isso precariza mais ainda os salários, diante das formas de vínculo empregatício que permitem longas jornadas de trabalho, sem tempo hábil para o descanso. Além disso, coloca a vida de milhares de trabalhadores da saúde em risco, diante da insalubridade de seus ambientes de trabalho. Posicionando-se no debate, o Conselho Federal de Enfermagem-COFEN ressalta que, especialmente, a MP $\mathrm{N}^{\circ} 927$ trata com desprezo os profissionais da saúde que batalham, diuturna e exaustivamente, no enfrentamento da COVID19 no Brasil (Cofen, 2020).

A MP No 927 desconsidera as diversas realidades, nos setores público e privado, que violentam estes profissionais, sobretudo os da Enfermagem, no que diz respeito ao subdimensionamento das equipes e à sobrecarga de trabalho, que já vinham sendo enfrentados por estes antes da COVID-19. O COFEN ressalta que jornadas exaustivas de trabalho estão relacionadas com o adoecimento dos profissionais e o aumento de risco de ocorrências adversas em serviços de saúde (Cofen, 2020).

A luta dos trabalhadores da saúde, no contexto da legislação flexibilizadora e da pandemia, é desalentadora, pois há, em muitos serviços, condições insalubres de trabalho, treinamento insuficiente e escassez de EPI. Além disso, o COFEN questiona o Art. 29 da MP No 927 que autoriza a dúvida, pelo empregador, da comprovação de nexo causal sobre casos de contaminação por COVID-19 em ambiente ocupacional (Cofen, 2020).

A revisão sistemática demonstrou que o tipo praticado de flexibilização do trabalho, a partir da MP $\mathrm{N}^{\circ} 927$, tende a apresentar indicadores maiores para acidentes e mortes devido ao trabalho, assim como o adoecimento em $90 \%$ dos que atuam sob a regência de tal normativa e congêneres (Druck, Dutra \& Silva, 2019). 
Para tanto, estes trabalhadores passam a vivenciar o trabalho como algo negativo, devido aos efeitos deletérios à saúde psicossocial, para a qual não vêem apoio, valorização ou reconhecimento (Lourenço et al, 2019). Desta forma, as leis e as medidas implementadas tendem a proteger somente o empregador, pois as promessas de resguardar o emprego se distanciam da garantia do direito à seguridade social trabalhista.

As medidas de flexibilização levam à precarização da proteção do trabalho e do trabalhador, observando-se um novo ciclo de concentração de riqueza, pois o marco legal implantado, atualmente, pelo Governo Federal brasileiro opera os direitos dos trabalhadores por meio da eliminação dos marcos legais que antes, na vigência plena da CLT, protegiam e asseguravam, estando, portanto, a reduzir cada vez mais as responsabilidades legais do empresariado (Filgueiras, Lima \& Souza, 2019).

Parece-nos que o Brasil não vem encontrando, em suas lideranças trabalhistas a força para enfrentar a conjuntura política liderada pelo Executivo brasileiro, enquanto a precarização das condições de trabalho enraíza-se por todos os setores da economia, empobrecendo e adoecendo os trabalhadores.

\subsection{Repercussões da medida provisória 927 de 2020 na saúde dos profissionais de saúde}

É sabido que trabalhadores de saúde sofrem uma sobrecarga inerente à natureza de seu ofício, sendo uma população comumente vulnerável ao adoecimento mental (Gold, 2020; Weintraub et al, 2020). Tal vulnerabilidade vem sendo historicamente negligenciada no Brasil, processo agudizado pela progressiva legitimação da precarização do trabalho, desde 2015 (Galvão et al, 2019), aprofundando-se em 2020 (Brasil, 2020), a partir da MP No 927.

Observa-se, no contexto, a redução relativa de sofrimentos físicos em comparação aos mentais, crescendo a emergência de quadros de ansiedade grave, comportamento depressivo e estafa laboral. Este processo de adoecimento é sintônico com a nova forma de trabalho, de produção do capital, seguindo o modelo de economia global que fragiliza os direitos trabalhistas, exacerbando o sentimento de insegurança e a vulnerabilidade do trabalhador (Santos et al, 2020).

Isso afeta, inclusive, a percepção e a sensação de sentimentos e afetos inerente às relações humanas, seja em família ou em sociedade, devido ao esgotamento mental que o processo produtivo precário acarreta (Han, 2015; Johnson, SalettiCuesta \& Tumas, 2020). Todos esses agravos à saúde mental vêm sendo intensificados pela pandemia do novo coronavírus, a qual vem expondo os profissionais de saúde a uma situação de constante sobrecarga laboral e emocional (Onu, 2020; Santos et al, 2020; Wang et al, 2020; Weintraub et al, 2020).

É sabido que a qualidade da saúde mental, em profissionais da saúde em situações de crise sanitária por epidemias ou pandemias, como já vivenciado nas crises da Síndrome Respiratória Aguda Grave-SARS, do Ebola e da H1N1, é deteriorada de modo intensivo (Wang et al, 2020). Tais crises tendem a colocar estes trabalhadores em um nível de tensão emocional alto, devido às medidas de segurança e saúde ocupacional, à necessidade de resposta da sociedade frente à doença, além do medo de propagar a infecção dentro do núcleo familiar e/ou pessoal (Faro et al, 2020; Who, 2020).

A tensão emocional tende a se agravar com as medidas de isolamento social e com o exercício de funções em serviços de saúde que apresentem alto risco de contaminação e exposição ao novo coronavírus. Esse processo constante de vigília tende comumente a provocar sintomas de exaustão, ansiedade, irritabilidade, insônia, déficit de concentração e queda do rendimento no trabalho (Brooks et al, 2020; Meira et al, 2020; Ornell et al, 2020). Embora sem estudos comparativos refinados, já se conhece a fotografia da situação atual em muitos países, a exemplo da China, onde 50,3\% dos trabalhadores de saúde da China relatam sintomas de depressão, 44,6\%, ansiedade e 34\%, de insônia (Gold, 2020).

No cenário brasileiro, os profissionais têm experimentado com maior intensidade esses problemas, devido ao aumento das jornadas de trabalho e à não obrigatoriedade sobre o fornecimento dos EPI pelos empregadores, devido à MP No 927 (Brasil, 2020; Fiho et al, 2020). A carga horária é tão intensa, com poucos momentos de descanso, que dificulta o simples ato de realizar as necessidades fisiológicas, para além do desconforto físico resultante da intensidade (Machado et al, 2020). 
Muitos destes profissionais precisam lidar com a ausência, insuficiência ou inadequação dos EPI (Fiho et al, 2020), o que reforça o estresse constante relacionado ao medo de contaminação (Faro, 2020; Onu, 2020; Who, 2020).

Além disso, enfrentam grandes barreiras diárias diante da ausência de gerência técnica do Ministério da Saúde, órgão máximo, necessário e estratégico, mas que demonstra falta de positividade nas ações, inclusive na elaboração e execução do Plano Nacional de Imunização. Urge enfrentarmos a necessidade de ações efetivas de preservação da vida e da qualidade de vida dos trabalhadores da saúde que estão vendo o número de infectados e óbitos aumentarem dia após dia, inclusive entre eles (Meira et al, 2020; Ornell et al, 2020).

Vale ressaltar que a exaltação midiática destes profissionais como super heróis traz consigo a mensagem subliminar de que precisam ser infalíveis, incansáveis, de que não podem adoecer ou fraquejar. E, contraditoriamente, os mesmos super poderes não vêm sendo reconhecidos na prática do apoio aos serviços prestados (Meira et al, 2020). Um claro exemplo dessa precarização é o fato de centenas de profissionais de saúde estarem atualmente com salários atrasados após terem atuado desde o início da pandemia da COVID-19 no Brasil (Bbc, 2020).

Evidencia-se que o futuro do trabalho no setor saúde, no Brasil, vem evoluindo para cenários cada vez mais precários, agregando riscos, antigos e novos, nos campos biológico, químico, ergonômico e psicossocial. É preciso problematizar a MP $\mathrm{N}^{\circ} 927$ pelo seu efetivo favorecimento à precariedade do trabalho, sobretudo no campo da saúde, favorecendo o adoecimento destes trabalhadores por meio da fragilização das condições de trabalho e acarretando perdas não só para os profissionais, como também para os usuários do sistema de saúde público e privado. Urge haver uma conjuntura política que busque defender e resguardar a proteção social trabalhista.

\section{Considerações Finais}

A pandemia da COVID-19, no Brasil, vem servindo de cenário para manobras que buscam precarizar o trabalho, o da saúde em particular, de forma mais ou menos velada, enquanto grande parte das lideranças trabalhistas, sindicais e grupos sociais estão preocupados com o enfrentamento da crise vivida pelo SUS, em decorrência da pandemia que agudiza desinvestimentos anteriores.

É a partir do caos gerado pelo novo coronavírus que o governo brasileiro vem tentando impor medidas provisórias, amparadas na reforma trabalhista de 2017, as quais retiram ou reduzem os direitos à segurança, à saúde e à assistência social. Agregando prejuízos, por sua vez, aos profissionais da saúde que estão se arriscando no enfrentamento à COVID-19.

É imprescindível que o Ministério Público do Trabalho, as associações sindicais e os demais órgãos que buscam a proteção do trabalhador e do trabalho reinventem e proponham novas formas para a organização da força trabalhista, a qual consiga lutar pela proteção dos direitos sociais trabalhistas, mesmo em tempos de crise pelo novo coronavírus.

Evidencia-se que o futuro do trabalho no setor saúde, no Brasil, vem evoluindo para cenários cada vez mais precários, agregando riscos, antigos e novos, nos campos biológico, químico, ergonômico e psicossocial. É preciso problematizar a MP № 927 pelo seu efetivo favorecimento à precariedade do trabalho, sobretudo no campo da saúde, favorecendo o adoecimento destes trabalhadores por meio da fragilização das condições de trabalho e acarretando perdas não só para os profissionais, como também para os usuários do sistema de saúde público e privado. Urge haver uma conjuntura política que busque defender e resguardar a proteção social trabalhista.

A força de trabalho em saúde ocupa lugar de destaque no cenário das soluções para as crises superpostas que foram descritas, sendo importante reconhecer as demandas presentes nesse cotidiano específico de trabalho. Procurou-se demonstrar que reflexões como estas são relevantes para ressignificar as repercussões na saúde mental do trabalhador, para o fortalecimento do trabalho, para a garantia de proteção social ocupacional e para o incremento de qualidade do funcionamento do SUS brasileiro, consequentemente da qualidade de vida da população. 


\section{Referências}

Andrade, C. B., Casulo, A. C. \& Alves G. (2018). Precarização do trabalho e saúde mental: o Brasil da Era Neoliberal. Bauru: Projeto Editorial Praxis, 1396.

Brasil. (2020). Presidência da República. Medida provisória $n^{o}$ 927, de 22 de março de 2020. Brasília: Presidência da República. http://www.in.gov.br/en/web/dou/-/medida-provisoria-n-927-de-22-de-marco-de-2020-249098775

British Broadcasting Corporation Brazil. BBC. (2020). “Antes, aplaudiam”: os profissionais da linha de frente da covid-19 que enfrentam atraso de salário. https://www.bbc.com/portuguese/brasil-53572522

Brooks, S. K., Webster, R. K., Smith, L. E., Woodland, L., Wessely, S., Greenberg, N. \& Rubin, G. J. (2020). The psychological impact of quarantine and how to reduce it: rapid review of the evidence. Lancet. 395: 912-20. https://www.thelancet.com/journals/lancet/article/PIIS0140-6736(20)30460-8/fulltext

Conselho Federal de Enfermagem do Brasil. (2020). COFEN vai à Justiça contra a Medida Provisória 927. COFEN, 2020. http://www.cofen.gov.br/cofenvai-a-justica-contra-medida-provisoria-927_78489.html

Central Única dos Trabalhadores do Brasil. (2020). Procurador do MPT critica Medida Provisória $n^{\circ} 927$ que impede atuação de sindicatos. CUT. https://www.cut.org.br/

Druck, G., Dutra, R. \& Silva, S. C. (2019). A contrarreforma neoliberal e a terceirização: a precarização como regra. Cad. CRH, 32 (86), 289-306. http://www.scielo.br/scielo.php?script=sci_arttext\&pid=S0103-49792019000200289\&lng=en\&nrm=iso

Faro, A., Bahiano, M. A., Nakano, T. C., Reis, C., Silva, B. F. P. \& Vitti, L. S. (2020). COVID-19 e saúde mental: a emergência do cuidado. Estud. psicol. (Campinas), Campinas, v. 37, e200074. https://doi.org/10.1590/1982-0275202037e200074

Fiho, J. M. J., Assunção, A. Á., Algranti, E., Garcia, E. G. \& Saito, C. A. (2020). A saúde do trabalhador e o enfrentamento da COVID-19. Rev. bras. saúde ocup., 45: e14. https://doi.org/10.1590/2317-6369ed0000120.

Filgueiras, V. A., Lima, U. M., Souza, I. F. (2019). Os impactos jurídicos, econômicos e sociais das reformas trabalhistas. Cad. CRH, 32(86): 231-252. http://dx.doi.org/10.9771/ccrh.v32i86.30731.

Gold, J. A. (2020). Covid-19: adverse mental health outcomes for healthcare workers. Editorials. BMJ, 369: m1815. 10.1136/bmj.m1815. https://doi.org/10.1136/bmj.m1815

Galvão, A. \& Marcelino, P. (2018). O sindicalismo brasileiro diante do golpe. In: Da Silva, S. G. C. et al. (Org.) Reformas institucionais de austeridade, democracia e relações de trabalho. LTr: p. 85-96.

Galvão, A., Castro, B., Krein, J. D. \& Teixeira, M. O. (2019). Reforma Trabalhista: precarização do trabalho e os desafios para o sindicalismo. Cad CRH. 32(86):253-269. https://doi.org/10.9771/ccrh.v32i86.30691

Galvão, A., Krein, J. D., Biavaschi, M. B. \& Teixeira, M. O. (2017). Contribuição Crítica à Reforma Trabalhista. Unicamp/Cesit. http://www.cesit.net.br/wpcontent/uploads/2017/06/

Han, B. C. (2015). Sociedade do Cansaço. Editora Vozes.

Johnson, M. C., Saletti-Cuesta, L. \& Tumas, N. (2020). Emoções, preocupações e reflexões diante da pandemia do Covid-19 na Argentina. Ciência \& saúde coletiva. Jun 25 (1), 2447-2456. https://doi.org/10.1590/1413-81232020256.1.10472020

Lourenço, E., Goulart, P., Anunciação, L. \& Lacaz, F. A. C. (2019). Condições de trabalho de assistentes sociais da área da saúde e repercussões psicossociais. Saúde soc. 28(1): 154-168. http://www.scielo.br/ scielo.php?script=sci_arttext\&pid=S0104-12902019000100011\&lng=en

Machado, W. C. A, Figueiredo, N. M. A., Brasil, S. S., Quaresma, M. L. J., Bittencourt, L. P., Tonini, T. \& Silva, P. S. (2020). Covid-19 in the paramentation movements of dressing and disposing of nurses: nightingale, the pioneer, was reasoned! Research, Society and Development, 9(7):1-23, e741974731. http://dx.doi.org/10.33448/rsd-v9i7.4731

Meira, K. C., Myrrha, L. J. D., Jesus, K. C., Oliveira, J. S. A., Silva, P. S. et al. (2020). O perfil e a sobrecarga na jornada de trabalho de profissionais da linha de frente ao combate à pandemia da COVID-19. ONAS-COVID-19. https://demografiaufrn.net/2020/05/13/sobrecarga-linha-frente/

Meneghetti, F. K. (2011). O que é um ensaio-teórico? Revista de Administração Contemporânea, 15(2), 320-332. https://doi.org/10.1590/S141565552011000200010

Menezes, A. P. R., Moretti, B. R. \& Reis, A. A. (2020). O futuro do SUS: impactos das reformas neoliberais na saúde pública - austeridade versus universalidade. Saúde em Debate. 43(5), 58-70. https://www.scielosp.org/article/sdeb/2019.v43nspe5/58-70/en/\#

ONU. (2020). Organização das Nações Unidas [Internet]. Agência de Notícias da Organização das Nações Unidas. Covid-19: OMS divulga guia com cuidados para saúde mental durante pandemia. OMS. https://news.un.org/pt/story/2020/03/1707792

Ornell, F., Halpern, S. C., Kessler, F. \& Narvaez, J. (2020). O impacto da pandemia de COVID-19 na saúde mental de profissionais de saúde. Cadernos de Saúde Pública. 36 (4), e00063520. https://doi.org/10.1590/0102-311X00063520

Passos, S. S., Lupatini, M. (2020). A contrarreforma trabalhista e a precarização das relações de trabalho no Brasil. Rev. katálysis, 23(1): 132-142. http://www.scielo.br/scielo.php?script=sci_arttext\&pid=S1414-49802020000100132\&lng=en\&nrm=iso

Pochmann, M. (2020). Tendências selecionadas para o mundo do trabalho no Brasil. Ciênc. saúde coletiva, 25 (1): 89-99. http://www.scielo.br/scielo.php?script=sci_arttext\&pid=S1413-81232020000100 089\&lng=en. 
Research, Society and Development, v. 10, n. 4, e27510414141, 2021

(CC BY 4.0) | ISSN 2525-3409 | DOI: http://dx.doi.org/10.33448/rsd-v10i4.14141

Santos, C. F. (2020). Reflexões sobre o impacto da pandemia de SARS-COV-2 / COVID-19 na saúde mental. Revista Brasileira de Psiquiatria. 42 (3), 329. https://doi.org/10.1590/1516-4446-2020-0981

Sintaema. (2020). Passar a boiada - Um ministro que parece não gostar do meio ambiente. https://sintaemasp.org.br/noticias/passar-a-boiada-um-ministroque-parece-nao-gostar-do-meio-ambiente

Wang, C., Pan, R., Wan, X., Tan, Y., Xu, L. Ho, C. S. et al. (2020). Immediate psychological responses and associated factors during the initial stage of the 2019 Coronavirus Disease (COVID-19) epidemic among the general population in China. International Journal of Environmental Research and Public Health. 17(5) 1729. https://www.mdpi.com/1660-4601/17/5/1729

Weintraub, A. C., Silva, A. C., Melo, B. D., Lima, C. C., Barbosa, C., Pereira, D. et al. (2020). Saúde mental e atenção psicossocial na pandemia COVID-19: orientações aos trabalhadores dos serviços de saúde. Fiocruz/CEPEDES.

World Health Organization (WHO). (2020). Coronavirus disease (COVID-19) outbreak: rights, roles and responsibilities of health workers, including key considerations for occupational safety and health. https://www.who.int/docs/default-source/coronaviruse/who-rights-roles-respon-hw-covid19.pdf?sfvrsn=bcabd401_0 